\title{
Algebras assigned to ternary relations
}

Ivan Chajda, Miroslav Kolařik, and Helmut Länger 


\title{
ALGEBRAS ASSIGNED TO TERNARY RELATIONS
}

\author{
IVAN CHAJDA, MIROSLAV KOLAŘÍK, AND HELMUT LÄNGER
}

Received 19 March, 2012

\begin{abstract}
We show that to every centred ternary relation $T$ on a set $A$ there can be assigned (in a non-unique way) a ternary operation $t$ on $A$ such that the identities satisfied by $(A ; t)$ reflect relational properties of $T$. We classify ternary operations assigned to centred ternary relations and we show how the concepts of relational subsystems and homomorphisms are connected with subalgebras and homomorphisms of the assigned algebra $(A ; t)$. We show that for ternary relations having a non-void median can be derived so-called median-like algebras $(A ; t)$ which become median algebras if the median $M_{T}(a, b, c)$ is a singleton for all $a, b, c \in A$. Finally, we introduce certain algebras assigned to cyclically ordered sets.
\end{abstract}

2010 Mathematics Subject Classification: 08A02; 08A05

Keywords: ternary relation, betweenness, cyclic order, assigned operation, centre, median

In [2] and [3], the first and the third author showed that to certain relational systems $\mathcal{A}=(A ; R)$, where $A \neq \varnothing$ and $R$ is a binary relation on $A$, there can be assigned a certain groupoid $\mathscr{G}(A)=(A ; \circ)$ which captures the properties of $R$. Namely, we have $x \circ y=y$ if and only if $(x, y) \in R$. In these papers we worked with so-called directed relational systems, i. e. for all $x, y \in A$ we have

$$
U_{R}(x, y):=\{z \in A \mid(x, z),(y, z) \in R\} \neq \varnothing .
$$

We are inspired by the idea of assigning a groupoid (called directoid) to a directed poset. This idea has its origin in the paper [6] by J. Ježek and R. Quackenbush. Then some structural properties of the assigned groupoid $\mathcal{Y}(A)$ can be used for introducing certain structural properties of $\mathcal{A}=(A ; R)$; in particular, we introduced congruences, quotient relational systems and homomorphisms which are in accordance with the corresponding concepts in $\mathscr{E}(A)$.

Hence, there arises the natural question if a similar way can be used for ternary relational systems and algebras with one ternary relation. In a particular case, such a

Support of the research of the first and third author by ÖAD, Cooperation between Austria and Czech Republic in Science and Technology, grant No. CZ 01/2011, of the first author by the Project CZ.1.07/2.3.00/20.0051 Algebraic Methods in Quantum Logics and of the second author by the Project CZ.1.07/2.3.00/20.0060 International Center for Information and Uncertainty is gratefully acknowledged. 
correspondence exists. It is for the ternary relation "betweenness" and the so-called median algebras, see e.g. [1,5] or [11].

However, there exist also other useful ternary relations for which a similar construction is not already derived, in particular the so-called cyclic orders, see e.g. $[4,7,8]$ and [9].

Moreover, more general ternary relations were already investigated in [10] and [11] and hence our problem can be extended to a more general case than betweenness. However, to get a construction of a ternary operation, a certain restriction on the ternary relation is necessary.

In the following let $A$ denote a fixed arbitrary non-empty set.

\section{TERNARY OPERATIONS ASSIGNED TO TERNARY RELATIONS}

We introduce the following concepts:

Definition 1. Let $T$ be a ternary relation on $A$ and $a, b \in A$. The set

$$
Z_{T}(a, b):=\{x \in A \mid(a, x, b) \in T\}
$$

is called the centre of $(a, b)$ with respect to $T$. The ternary relation $T$ on $A$ is called centred if $Z_{T}(a, b) \neq \varnothing$ for all elements $a, b \in A$.

Definition 2. Let $T$ be a ternary relation on $A$ and $a, b, c \in A$. The set

$$
M_{T}(a, b, c):=Z_{T}(a, b) \cap Z_{T}(b, c) \cap Z_{T}(c, a)
$$

will be called the median of $(a, b, c)$ with respect to $T$.

The concept of a median was originally introduced in lattices and structures derived from lattices. In particular, two sorts of medians are usually considered: $m(x, y, z)=(x \wedge y) \vee(y \wedge z) \vee(z \wedge x)$ and $M(x, y, z)=(x \vee y) \wedge(y \vee z) \wedge(z \vee x)$.

Now we show that to every centred ternary relation there can be assigned ternary operations.

Definition 3. Let $T$ be a centred ternary relation on $A$ and $t$ a ternary operation on $A$ satisfying

$$
t(a, b, c) \begin{cases}=b & \text { if }(a, b, c) \in T \\ \in Z_{T}(a, c) & \text { otherwise. }\end{cases}
$$

Such an operation $t$ is called assigned to $T$.

Remark 1. By definition, if $T$ is a centred ternary relation on $A$ and $t$ assigned to $T$ then $(a, t(a, b, c), c) \in T$ for all $a, b, c \in A$.

Lemma 1. Let $T$ be a centred ternary relation on $A$ and $t$ an assigned operation. Let $a, b, c \in A$. Then $(a, b, c) \in T$ if and only if $t(a, b, c)=b$.

Proof. By Definition 3, if $(a, b, c) \in T$ then $t(a, b, c)=b$. Conversely, assume $(a, b, c) \notin T$. Then $t(a, b, c) \in Z_{T}(a, c)$. Now $t(a, b, c)=b$ would imply $(a, b, c)=$ $(a, t(a, b, c), c) \in T$ contradicting $(a, b, c) \notin T$. Hence $t(a, b, c) \neq b$. 
To illuminate the role of the median, let us consider the following example:

Example 1 . Let $\mathscr{L}=(L ; \vee, \wedge)$ be a lattice. Define a ternary operation $T$ on $L$ as follows:

$$
(a, b, c) \in T \quad \text { if and only if } \quad a \wedge c \leq b \leq a \vee c .
$$

Put $m(x, y, z):=(x \wedge y) \vee(y \wedge z) \vee(z \wedge x)$ and $M(x, y, z):=(x \vee y) \wedge(y \vee z) \wedge$ $(z \vee x)$. If $p \in M_{T}(a, b, c)$ then $p \in Z_{T}(a, b), p \in Z_{T}(b, c)$ and $p \in Z_{T}(c, a)$, i. e. $a \wedge b \leq p \leq a \vee b, b \wedge c \leq p \leq b \vee c$ and $c \wedge a \leq p \leq c \vee a$ whence $m(a, b, c) \leq p \leq$ $M(a, b, c)$. This yields

$$
M_{T}(a, b, c)=[m(a, b, c), M(a, b, c)],
$$

the interval in $\mathscr{L}$. It is well-known that $m(x, y, z)=M(x, y, z)$ if and only if $\mathscr{L}$ is distributive. Hence, $\mathscr{L}$ is distributive if and only if $\left|M_{T}(a, b, c)\right|=1$ for all $a, b, c \in$ $L$.

The previous example was used in [5] for the definition of a median algebra. If $\mathscr{L}$ is a distributive lattice then the algebra $(L ; m)$ is called the median algebra derived from $\mathscr{L}$. Of course, there exist median algebras which are not derived from a lattice, see [1] for details, but in every median algebra there can be introduced a ternary relation "between" by putting

$$
(a, b, c) \in T_{m} \quad \text { if and only if } \quad m(a, b, c)=b .
$$

In what follows, we show how this construction can be generalized and we get a characterization of some important properties of ternary relations by means of identities of their assigned operations.

Theorem 1. A ternary operation $t$ on $A$ is assigned to some centred ternary relation $T$ on $A$ if and only if it satisfies the identity

$$
t(x, t(x, y, z), z)=t(x, y, z) .
$$

Proof. Let $a, b, c \in A$.

Assume that $T$ is a ternary relation on $A$ and $t$ an assigned operation. If $(a, b, c) \in$ $T$ then $t(a, b, c)=b$ and hence $t(a, t(a, b, c), c)=t(a, b, c)$. If $(a, b, c) \notin T$ then $t(a, b, c) \in Z_{T}(a, c)$ and hence $(a, t(a, b, c), c) \in T$ which yields $t(a, t(a, b, c), c)=$ $t(a, b, c)$. Thus $t$ satisfies identity (1.1).

Conversely, assume $t: A^{3} \rightarrow A$ satisfies (1.1) and define $T:=$ $\left\{(x, y, z) \in A^{3} \mid t(x, y, z)=y\right\}$. If $(a, b, c) \in T$ then $t(a, b, c)=b$ and, if $(a, b, c) \notin T$ then $(a, t(a, b, c), c) \in T$ whence $t(a, b, c) \in Z_{T}(a, c)$, i. e. $t$ is assigned to $T$.

We can consider a number of properties of ternary relations which are used in [1-11] for "betweenness" and for "cyclic orders".

Definition 4. Let $T$ be a ternary relation on $A$. We call $T$ - reflexive if $|\{a, b, c\}| \leq 2$ implies $(a, b, c) \in T$;

- symmetric if $(a, b, c) \in T$ implies $(c, b, a) \in T$; 
- antisymmetric if $(a, b, a) \in T$ implies $a=b$;

- cyclic if $(a, b, c) \in T$ implies $(b, c, a) \in T$;

- $R$-transitive if $(a, b, c),(b, d, e) \in T$ implies $(a, d, e) \in T$;

- $t_{1}$-transitive if $(a, b, c),(a, d, b) \in T$ implies $(d, b, c) \in T$;

- $t_{2}$-transitive if $(a, b, c),(a, d, b) \in T$ implies $(a, d, c) \in T$;

$-R$-symmetric if $(a, b, c) \in T$ implies $(b, a, c) \in T$;

- $R$-antisymmetric if $(a, b, c),(b, a, c) \in T$ implies $a=b$;

- non-sharp if $(a, a, b) \in T$ for all $a, b \in A$;

- cyclically transitive if $(a, b, c),(a, c, d) \in T$ implies $(a, b, d) \in T$.

Theorem 2. Let $T$ be a centred ternary relation on $A$ and $t$ an assigned operation. Then $(i)-(x i)$ hold:

(i) $T$ is reflexive if and only if $t$ satisfies the identities

$$
t(x, x, y)=t(y, x, x)=t(y, x, y)=x .
$$

(ii) $T$ is symmetric if and only if t satisfies the identity

$$
t(z, t(x, y, z), x)=t(x, y, z) .
$$

(iii) $T$ is antisymmetric if and only if $t$ satisfies the identity

$$
t(x, y, x)=x .
$$

(iv) $T$ is cyclic if and only if $t$ satisfies the identity

$$
t(t(x, y, z), z, x)=z \text {. }
$$

(v) $T$ is $R$-transitive if and only if $t$ satisfies the identity

$$
t(x, t(t(x, y, z), u, v), v)=t(t(x, y, z), u, v) .
$$

(vi) $T$ is $t_{1}$-transitive if and only if $t$ satisfies the identity

$$
t(t(x, u, t(x, y, z)), t(x, y, z), z)=t(x, y, z) .
$$

(vii) $T$ is $t_{2}$-transitive if and only if t satisfies the identity

$$
t(x, t(x, u, t(x, y, z)), z)=t(x, u, t(x, y, z)) .
$$

(viii) $T$ is $R$-symmetric if and only if t satisfies the identity

$$
t(t(x, y, z), x, z)=x .
$$

(ix) If t satisfies the identity

$$
t(t(x, y, z), x, z)=t(x, y, z)
$$

then $T$ is $R$-antisymmetric.

(x) $T$ is non-sharp if and only if $t$ satisfies the identity

$$
t(x, x, y)=x .
$$

(xi) $T$ is cyclically transitive if and only if $t$ satisfies the identity

$$
t(x, t(x, y, t(x, z, u)), u)=t(x, y, t(x, z, u)) .
$$


Proof. Let $a, b, c, d, e \in A$.

(i) is clear.

(ii) $t$ satisfies $t(z, t(x, y, z), x)=t(x, y, z)$ if and only if $(z, t(x, y, z), x) \in T$ for all $x, y, z \in A$.

" $\Rightarrow ":(a, t(a, b, c), c) \in T$ and hence $(c, t(a, b, c), a) \in T$.

" $\Leftarrow$ ": If $(a, b, c) \in T$ then $(c, b, a)=(c, t(a, b, c), a) \in T$.

(iii) " $\Rightarrow ":(a, t(a, b, a), a) \in T$ and hence $t(a, b, a)=a$.

" $\Leftarrow$ ": If $(a, b, a) \in T$ then $a=t(a, b, a)=b$.

(iv) $t$ satisfies $t(t(x, y, z), z, x)=z$ if and only if $(t(x, y, z), z, x) \in T$ for all $x, y, z \in$ $A$.

" $\Rightarrow ":(a, t(a, b, c), c) \in T$ and hence $(t(a, b, c), c, a) \in T$.

" $\Leftarrow$ ": If $(a, b, c) \in T$ then $(b, c, a)=(t(a, b, c), c, a) \in T$.

(v) $t$ satisfies $t(x, t(t(x, y, z), u, v), v)=t(t(x, y, z), u, v)$ if and only if

$(x, t(t(x, y, z), u, v), v) \in T$ for all $x, y, z, u, v \in A$.

" $\Rightarrow ":(a, t(a, b, c), c),(t(a, b, c), t(t(a, b, c), d, e), e) \in T$ and hence

$(a, t(t(a, b, c), d, e), e) \in T$.

" $\Leftarrow$ ": If $(a, b, c),(b, d, e) \in T$ then $(a, d, e)=(a, t(t(a, b, c), d, e), e) \in T$.

(vi) $t$ satisfies $t(t(x, u, t(x, y, z)), t(x, y, z), z)=t(x, y, z)$ if and only if

$(t(x, u, t(x, y, z)), t(x, y, z), z) \in T$ for all $x, y, z, u \in A$.

" $\Rightarrow ":(a, t(a, b, c), c),(a, t(a, d, t(a, b, c)), t(a, b, c)) \in T$ and hence

$(t(a, d, t(a, b, c)), t(a, b, c), c) \in T$.

" $\Leftarrow$ ": If $(a, b, c),(a, d, b) \in T$ then $(d, b, c)=(t(a, d, t(a, b, c)), t(a, b, c), c) \in T$.

(vii) $t$ satisfies $t(x, t(x, u, t(x, y, z)), z)=t(x, u, t(x, y, z))$ if and only if

$(x, t(x, u, t(x, y, z)), z) \in T$ for all $x, y, z, u \in A$.

" $\Rightarrow ":(a, t(a, b, c), c),(a, t(a, d, t(a, b, c)), t(a, b, c)) \in T$ and hence

$(a, t(a, d, t(a, b, c)), c) \in T$.

" $\Leftarrow$ ": If $(a, b, c),(a, d, b) \in T$ then $(a, d, c)=(a, t(a, d, t(a, b, c)), c) \in T$.

(viii) $t$ satisfies $t(t(x, y, z), x, z)=x$ if and only if $(t(x, y, z), x, z) \in T$ for all $x, y, z \in$ $A$.

" $\Rightarrow$ ": $(a, t(a, b, c), c) \in T$ and hence $(t(a, b, c), a, c) \in T$.

" $\Leftarrow$ ": If $(a, b, c) \in T$ then $(b, a, c)=(t(a, b, c), a, c) \in T$.

(ix) If $(a, b, c),(b, a, c) \in T$ then $a=t(b, a, c)=t(t(a, b, c), a, c)=t(a, b, c)=b$.

(x) This is clear.

(xi) $t$ satisfies $t(x, t(x, y, t(x, z, u)), u)=t(x, y, t(x, z, u))$ if and only if

$(x, t(x, y, t(x, z, u)), u) \in T$ for all $x, y, z, u \in A$.

" $\Rightarrow ":(a, t(a, b, t(a, c, d)), t(a, c, d)),(a, t(a, c, d), d) \in T$ and hence

$(a, t(a, b, t(a, c, d)), d) \in T$.

" $\Leftarrow$ ": If $(a, b, c),(a, c, d) \in T$ then $t(a, b, d)=t(a, t(a, b, t(a, c, d)), d)$

$=t(a, b, t(a, c, d))=b$. 
Lemma 2. Let $T$ be a ternary relation on $A$. Then

$$
|T|=\sum_{(a, b) \in A^{2}}\left|Z_{T}(a, b)\right| .
$$

Proof.

$$
T=\bigcup_{(a, b) \in A^{2}}^{\bullet}\left(\{a\} \times Z_{T}(a, b) \times\{b\}\right) .
$$

Corollary 1. Let $A$ be finite, $|A|=n$. If $T$ is a centred ternary relation on $A$ then $|T| \geq n^{2}$. Moreover, if $T$ is centred then $|T|=n^{2}$ if and only if $\left|Z_{T}(x, y)\right|=1$ for each $x, y \in A$.

\section{CONGRUENCES, HOMOMORPHISMS AND SUBSYSTEMS OF TERNARY RELATIONAL SYSTEMS}

By a ternary relational system is meant a couple $\mathcal{T}=(A ; T)$ where $T$ is a ternary relation on $A$. $\mathcal{T}$ is called centred if $T$ is centred. As shown in the previous section, to every centred ternary relational system $\mathcal{T}=(A ; T)$ there can be assigned an algebra $\mathcal{A}(T)=(A ; t)$ with one ternary operation $t: A^{3} \rightarrow A$ such that $t$ is assigned to $T$. Now, we can introduce an inverse construction. It means that to every algebra $\mathcal{A}=$ $(A ; t)$ of type (3) there can be assigned a ternary relational system $\mathcal{T}(A)=\left(A ; T_{t}\right)$ where $T_{t}$ is defined by

$$
T_{t}:=\left\{(x, y, z) \in A^{3} \mid t(x, y, z)=y\right\} .
$$

Of course, an assigned ternary relational system $\mathcal{T}(A)=\left(A ; T_{t}\right)$ need not be centred. However, if $\mathcal{T}=(A ; T)$ is a centred ternary relational system and $\mathcal{A}(T)=(A ; t)$ an assigned algebra then $T_{t}$ is centred despite the fact that $t$ is not determined uniquely. In fact, we have $(a, b, c) \in T_{t}$ if and only if $t(a, b, c)=b$ if and only if $(a, b, c) \in T$. Hence, we have proved the following

Lemma 3. Let $\mathcal{T}=(A ; T)$ be a centred ternary relational system, $\mathcal{A}(T)=(A ; t)$ an assigned algebra and $\mathcal{T}(\mathcal{A}(T))=\left(A ; T_{t}\right)$ the ternary relational system assigned to $\mathcal{A}(T)$. Then $\mathcal{T}(\mathcal{A}(T))=\mathcal{T}$.

The best known correspondence between centred ternary relational systems and corresponding algebras of type (3) is the case of "betweenness"-relations and median algebras which was initiated by J. R. Isbell [5] and essentially developed by H.-J. Bandelt and J. Hedlíková [1]. However, there are also some essential differences between relational systems and the corresponding algebras. For binary relational systems it was described by the first and the third author in [2]. In what follows, we are going to handle it for the ternary case. 
If $\mathcal{T}=(A ; T)$ is a ternary relational system and $E$ an equivalence relation on $A$ then the quotient relational system $\mathcal{T} / E$ is defined as the relational system $(A / E, T / E)$ where $T / E:=\left\{\left([x]_{E},[y]_{E},[z]_{E}\right) \mid(x, y, z) \in T\right\}$. It is evident that $E$ need not be a congruence on the assigned algebra $\mathcal{A}(T)=(A ; t)$ and hence congruences on $\mathcal{T}=(A ; T)$, respectively on $\mathcal{A}(T)$ are different concepts.

Similarly, by a subsystem of $\mathcal{T}=(A ; T)$ is meant a couple of the form $(B, T \mid B)$ with a non-empty subset $B$ of $A$ and $T \mid B:=T \cap B^{3}$. One can easily see that this need not be a subalgebra of $\mathcal{A}(T)=(A ; t)$.

Finally, by a homomorphism of a ternary relational system $\mathcal{T}=(A ; T)$ into a ternary relational system $\delta=(B ; S)$ is meant a mapping $h: A \rightarrow B$ satisfying

$$
(a, b, c) \in T \quad \Longrightarrow \quad(h(a), h(b), h(c)) \in S .
$$

A homomorphism $h$ is called strong if for each triple $(p, q, r) \in S$ there exists $(a, b, c) \in T$ such that $(h(a), h(b), h(c))=(p, q, r)$.

Now, we define the following concept.

Definition 5. A $t$-homomorphism from a centred ternary relational system $\mathcal{T}=$ $(A ; T)$ to a ternary relational system $\delta=(B ; S)$ is a homomorphism from $\mathcal{T}$ to $\delta$ such that there exists an algebra $(A ; t)$ assigned to $\mathcal{T}$ such that $a, b, c, a^{\prime}, b^{\prime}, c^{\prime} \in A$ and $(h(a), h(b), h(c))=\left(h\left(a^{\prime}\right), h\left(b^{\prime}\right), h\left(c^{\prime}\right)\right)$ together imply $h(t(a, b, c))=h\left(t\left(a^{\prime}, b^{\prime}, c^{\prime}\right)\right)$.

Theorem 3. Let $\mathcal{T}=(A ; T)$ and $\delta=(B ; S)$ be centred ternary relational systems and $\mathcal{A}(T)=(A ; t)$ and $\mathscr{B}(S)=(B ; s)$ assigned algebras. Then every homomorphism from $\mathcal{A}(T)$ to $\mathcal{B}(S)$ is a t-homomorphism from $\mathcal{T}$ to 8 .

Proof. Let $a, b, c, a^{\prime}, b^{\prime}, c^{\prime} \in A$. If $(a, b, c) \in T$ then $t(a, b, c)=b$ and hence $s(h(a), h(b), h(c))=h(t(a, b, c))=h(b)$ showing $(h(a), h(b), h(c)) \in S$. Thus $h$ is a homomorphism from $\mathcal{T}$ to $\delta$.

Moreover, if $(h(a), h(b), h(c))=\left(h\left(a^{\prime}\right), h\left(b^{\prime}\right), h\left(c^{\prime}\right)\right)$ then

$$
h(t(a, b, c))=s(h(a), h(b), h(c))=s\left(h\left(a^{\prime}\right), h\left(b^{\prime}\right), h\left(c^{\prime}\right)\right)=h\left(t\left(a^{\prime}, b^{\prime}, c^{\prime}\right)\right) .
$$

Hence $h$ is a $t$-homomorphism from $\mathcal{T}$ to $\delta$.

The theorem just proved says that every homomorphism of assigned algebras is a $t$-homomorphism of the original relational systems. Now we can show under which conditions the converse assertion becomes true.

Theorem 4. Let $\mathcal{T}=(A ; T)$ and $\delta=(B ; S)$ be centred ternary relational systems. Then for every strong $t$-homomorphism $h$ from $\mathcal{T}$ to $\&$ with assigned algebra $\mathcal{A}(T)=(A ; t)$ there exists an algebra $\mathscr{B}(S)=(B ; s)$ assigned to 8 such that $h$ is a homomorphism from $\mathcal{A}(T)$ to $\mathcal{B}(S)$.

Proof. Let $h$ be a strong $t$-homomorphism from $\mathcal{T}$ to $\delta$. By definition there exists an algebra $\mathcal{A}(T)=(A ; t)$ assigned to $\mathcal{T}$ such that for all $a, b, c, a^{\prime}, b^{\prime}, c^{\prime} \in A$ with 
$(h(a), h(b), h(c))=\left(h\left(a^{\prime}\right), h\left(b^{\prime}\right), h\left(c^{\prime}\right)\right)$ it holds $h(t(a, b, c))=h\left(t\left(a^{\prime}, b^{\prime}, c^{\prime}\right)\right)$. Define a ternary operation $s$ on $B$ as follows:

$$
s(h(x), h(y), h(z)):=h(t(x, y, z))
$$

for all $x, y, z \in A$. Since $h$ is strong and a $t$-homomorphism, $s$ is correctly defined. For $a, b, c \in A$, if $(h(a), h(b), h(c)) \in S$ then there exists $(d, e, f) \in T$ such that $(h(d), h(e), h(f))=(h(a), h(b), h(c))$. Now

$$
s(h(a), h(b), h(c))=h(t(a, b, c))=h(t(d, e, f))=h(e)=h(b) .
$$

If $(h(a), h(b), h(c)) \notin S$ then $(a, b, c) \notin T$ since $h$ is a homomorphism from $\mathcal{T}$ to $\delta$ and hence $t(a, b, c) \in Z_{T}(a, c)$, i. e. $(a, t(a, b, c), c) \in T$. Thus $(h(a), h(t(a, b, c)), h(c)) \in S$, i. e.

$$
(h(a), s(h(a), h(b), h(c)), h(c)) \in S
$$

whence $s(h(a), h(b), h(c)) \in Z_{S}(h(a), h(c))$. This shows that $\mathscr{B}(S)$ is an algebra assigned to $\mathscr{B}$. It is easy to see that $h$ is a homomorphism from $\mathcal{A}(T)$ to $\mathscr{B}(S)$.

We are going to get connections between $t$-homomorphisms of relational systems and congruences on the assigned algebras.

Theorem 5. Let $\mathcal{T}=(A ; T), \&=(B ; S)$ be centred ternary relational systems. Then the following hold:

(i) If $h$ is a strong $t$-homomorphism from $\mathcal{T}$ to $\&$ then there exists an algebra $\mathcal{A}(T)=(A ; t)$ assigned to $\mathcal{T}$ such that $\operatorname{ker} h \in \operatorname{Con} \mathcal{A}(T)$.

(ii) If $\mathcal{A}(T)=(A ; t)$ is an algebra assigned to $\mathcal{T}$ and $\theta \in \operatorname{Con} \mathcal{A}(T)$ then the canonical mapping $h: A \rightarrow A / \theta$ is a strong $t$-homomorphism from $\mathcal{T}$ onto $\mathcal{T} / \theta$.

Proof. (i) Let $h$ be a strong $t$-homomorphism from $\mathcal{T}$ to $\delta$. By definition and Theorem 4, there exist assigned algebras $\mathcal{A}(T)=(A ; t)$, respectively $\mathscr{B}(S)=(B ; s)$ such that $h$ is a homomorphism of $\mathcal{A}(T)$ to $\mathscr{B}(S)$ and hence ker $h \in \operatorname{Con} \mathcal{A}(T)$.

(ii) Let $\mathcal{A}(T)=(A ; t)$ be an algebra assigned to $\mathcal{T}, \theta \in \operatorname{Con} \mathcal{A}(T)$ and $h: A \rightarrow$ $A / \theta$ denote the canonical mapping. By definition of $T / \theta$, if $(a, b, c) \in T$ then $(h(a), h(b), h(c)) \in T / \theta$ and hence $h$ is a homomorphism from $\mathcal{T}$ to $\mathcal{T} / \theta$. If, moreover, $a, b, c, a^{\prime}, b^{\prime}, c^{\prime} \in A$ and $(h(a), h(b), h(c))=\left(h\left(a^{\prime}\right), h\left(b^{\prime}\right), h\left(c^{\prime}\right)\right)$ then

$$
h(t(a, b, c))=t(h(a), h(b), h(c))=t\left(h\left(a^{\prime}\right), h\left(b^{\prime}\right), h\left(c^{\prime}\right)\right)=h\left(t\left(a^{\prime}, b^{\prime}, c^{\prime}\right)\right) .
$$

Therefore $h$ is a $t$-homomorphism from $\mathcal{T}$ onto $\mathcal{T} / \theta$. Obviously, $h$ is strong.

Definition 6. Let $\mathcal{T}=(A ; T)$ be a centred ternary relational system. An equivalence relation $\theta$ on $A$ is called a $t$-congruence on $\mathcal{T}$ if there exists an algebra $\mathcal{A}(T)=(A ; t)$ assigned to $\mathcal{T}$ such that $\theta \in \operatorname{Con} \mathcal{A}(T)$. A subset $B$ of $A$ is called a $t$-subsystem of $\mathcal{T}$ if there exists an algebra $\mathcal{A}(T)=(A ; t)$ assigned to $\mathcal{T}$ such that $(B ; t)$ is a subalgebra of $\mathcal{A}(T)$. 
Example 2. Consider $A=\{a, b, c, d\}$ and the ternary relation $T$ on $A$ defined as follows: $T:=A \times\{d\} \times A$. Then $d \in Z_{T}(x, y)$ for each $x, y \in A$ and hence $T$ is centred and its median is non-empty, in fact $M_{T}(x, y, z)=\{d\}$ for all $x, y, z \in A$. For $B=\{a, b, c\}, \mathscr{B}=(B ; T \mid B)$ is a subsystem of $\mathcal{A}=(A ; T)$ but it is not a $t$ subsystem. Namely, for every $x, y, z \in A t$ can be defined in the unique way as follows: $t(x, y, z):=d$. Hence, $(\{a, b, c\} ; t)$ is not a subalgebra of $(A ; t)$. On the contrary, $\{a, b, d\},\{a, c, d\},\{b, c, d\}$ are $t$-subsystems of $\mathcal{A}$.

Remark 2. Let $\mathcal{A}=(A ; t), \mathscr{B}=(B ; s)$ be algebras of type (3) and $h: A \rightarrow B$ a homomorphism from $\mathcal{A}$ to $\mathcal{B}$. Put $\mathcal{T}(A):=\left(A ; T_{t}\right)$ and $\mathcal{S}(B):=\left(B ; S_{S}\right)$ where $T_{t}$, $S_{s}$ are defined by (2.1). Then $h$ need not be a $t$-homomorphism of $\mathcal{T}(A)$ to $\delta(B)$, see the following example.

Example 3. Let $A=\{-1,0,1\}, B=\{1,0\}$ and $t(x, y, z)=x \cdot y, s(x, y, z)=x \cdot y$, where "." is the multiplication of integers. Let $h: A \rightarrow B$ be defined by $h(x)=|x|$. Then $h$ is clearly a homomorphism from $\mathcal{A}=(A ; t)$ to $\mathcal{B}=(B ; s)$ and

$$
T_{t}=(A \times\{0\} \times A) \cup\left(\{1\} \times A^{2}\right) .
$$

There exists exactly one algebra $\left(A ; t^{*}\right)$ assigned to $\mathcal{T}(A)$, namely where

$$
t^{*}(x, y, z):= \begin{cases}y & \text { if } y=0 \text { or } x=1 \\ 0 & \text { otherwise. }\end{cases}
$$

Now $h(-1)=h(1)$ but $h\left(t^{*}(-1,-1,1)\right)=h(0)=0 \neq 1=h(1)=h\left(t^{*}(1,1,1)\right)$. Thus $h$ is not a $t$-homomorphism.

We can prove the following:

Theorem 6. If $\mathcal{A}=(A ; t)$ and $\mathcal{B}=(B ; s)$ are algebras of type $(3)$, $\mathcal{A}$ satisfies the identity

$$
t(x, t(x, y, z), z)=t(x, y, z)
$$

and $\mathcal{T}(A)=\left(A ; T_{t}\right)$ and $\delta(B)=\left(B ; S_{S}\right)$ denote the relational systems corresponding to $\mathcal{A}$ and $\mathcal{B}$, respectively, as defined by (2.1) then every homomorphism $h$ from $\mathcal{A}$ to $\mathcal{B}$ is a $t$-homomorphism from $\mathcal{T}(A)$ to $\mathcal{S}(B)$.

Proof. Let $a, b, c, d, e, f \in A$. If $(a, b, c) \in \mathcal{T}(A)$ then $t(a, b, c)=b$ and hence

$$
s(h(a), h(b), h(c))=h(t(a, b, c))=h(b)
$$

whence $(h(a), h(b), h(b)) \in \delta(B)$. This shows that $h$ is a homomorphism from $\mathcal{T}(A)$ to $\delta(B)$. Obviously, $t$ is assigned to $T_{t}$. Finally, $(h(a), h(b), h(c))=(h(d), h(e), h(f))$ implies

$$
h(t(a, b, c))=s(h(a), h(b), h(c))=s(h(d), h(e), h(f))=h(t(d, e, f))
$$

which shows that $h$ is a $t$-homomorphism from $\mathcal{T}(A)$ to $\delta(B)$. 


\section{DERIVED BINARY SYSTEMS}

Let $T$ be a ternary relation on $A$ and $p$ an arbitrary, but fixed element of $A$. Then

$$
R_{T}:=\left\{(x, y) \in A^{2} \mid(x, y, p) \in T\right\}
$$

is called the binary relation $p$-derived from T. Moreover, put $x \circ y:=t(x, y, p)$ for all $x, y \in A$ if $T$ is centred and $t$ is an assigned operation.

If $T$ is reflexive then $R_{T}$ is reflexive, too. If, moreover, $T$ is centred then Theorem 2 implies $x \circ x=x$, the idempotency of the operation $\circ$ which is in accordance with (i) of Theorem 8 in [3].

Similarly, if $T$ is $R$-symmetric then $R_{T}$ is symmetric. If, moreover, $T$ is centred then Theorem 2 implies $(x \circ y) \circ x=x$ which is identity (ii) of Theorem 8 in [3] characterizing symmetric binary relations (for directed relational systems).

If $T$ is $R$-antisymmetric then $R_{T}$ is antisymmetric. If, moreover, $T$ is centred then Theorem 2 yields that $(x \circ y) \circ x=x \circ y$ which, if satisfied for all $p \in A$, is a sufficient condition for the antisymmetry of $R_{T}$. This condition is also a sufficient condition for the antisymmetry of binary relations (see (v) of Theorem 8 in [3]).

If $T$ is $R$-transitive then $R_{T}$ is transitive. If, moreover, $T$ is centred then Theorem 2 implies $x \circ((x \circ y) \circ u)=(x \circ y) \circ u$ which is just identity (iii) of Theorem 8 in [3] characterizing transitivity of binary relations.

Let us recall from [3] that a binary relation $R$ on $A$ is (upward) directed if

$$
U_{R}(a, b):=\{x \in A \mid(a, x),(b, x) \in R\} \neq \varnothing \text { for all } a, b \in A .
$$

Although reflexivity, $R$-symmetry, $R$-antisymmetry and $R$-transitivity of a ternary relation $T$ on $A$ yields the corresponding property of $R_{T}$, we are not able to show that if $T$ is centred then $R_{T}$ is directed. However, our characterization of the corresponding properties for binary relations by means of the induced binary operations in [3] are possible for directed relations only.

Example 4. Put $A:=\{x, y, z\}$ and

$$
T:=\{(x, z, y)\} \cup\left\{(a, y, b) \mid(a, b) \in A^{2} \backslash\{(x, y)\}\right\} .
$$

Then $T$ is centred because $Z_{T}(x, y)=\{z\}$ and $Z_{T}(a, b)=\{y\}$ for $(a, b) \in A^{2} \backslash$ $\{(x, y)\}$. Put $p:=y$ and consider the $p$-derived binary relation $R_{T}$ on $A$. Then

$$
x \circ(x \circ y)=t(x, t(x, y, y), y)=t(x, z, y)=z=t(x, y, y)=x \circ y,
$$

but

$$
y \circ(x \circ y)=t(y, t(x, y, y), y)=t(y, z, y)=y \neq z=t(x, y, y)=x \circ y .
$$

Thus $y \circ(x \circ y) \neq(x \circ y)$. According to (ii) of Theorem 6 in [3], $R_{T}$ is not directed.

Remark 3. Theorem 6 in [3] says that for a groupoid $(G ; \circ)$ the following are equivalent: 
(i) There exists a directed relational system $(G ; R)$ with a reflexive relation $R$ such that $(G ; \circ)$ corresponds to $(G ; R)$.

(ii) $(G ; \circ)$ satisfies the identities $x \circ x=x$ and $x \circ(x \circ y)=y \circ(x \circ y)=x \circ y$.

We are going to show a sufficient condition for $R_{T}$ to be directed.

Theorem 7. Let $T$ be a reflexive ternary relation on $A$ such that $Z_{T}(a, c) \cap$ $Z_{T}(b, c) \neq \varnothing$ for all $a, b, c \in A$. Let $p \in A$ and $R_{T}$ denote the binary relation $p$-derived from $T$. Then $R_{T}$ is directed.

Proof. Due to the assumption, $T$ is centred and hence we can consider a ternary operation $t$ on $A$ assigned to $T$ such that $t(a, b, c) \in Z_{T}(a, c) \cap Z_{T}(b, c)$ if $(a, b, c) \in$ $A^{3} \backslash T$. Since $T$ is reflexive, we have $x \circ x=t(x, x, p)=x$.

First assume $(x, y) \in R_{T}$. Then $(x, y, p) \in T$. Thus $t(x, y, p)=y$ and hence

$$
x \circ(x \circ y)=t(x, t(x, y, p), p)=t(x, y, p)=x \circ y .
$$

Since $T$ is reflexive, we obtain

$$
y \circ(x \circ y)=t(y, t(x, y, p), p)=t(y, y, p)=y=t(x, y, p)=x \circ y .
$$

Now suppose $(x, y) \notin R_{T}$. Then

$$
x \circ(x \circ y)=t(x, t(x, y, p), p)=t(x, y, p)=x \circ y .
$$

Since $t(x, y, p) \in Z_{T}(x, p) \cap Z_{T}(y, p)$ we have also $t(x, y, p) \in Z_{T}(y, p)$ and hence

$$
y \circ(x \circ y)=t(y, t(x, y, p), p)=t(x, y, p)=x \circ y .
$$

We have shown that o satisfies (ii) of Theorem 6 in [3]. Thus $R_{T}$ is directed.

The converse assertion is also true. For a binary relation $R$ on $A$ and a fixed element $p \in A$ we define

$$
T_{p}(R):=\{(x, y, p) \mid(x, y) \in R\} \cup\{(x, x, y) \mid x, y \in A\} .
$$

Then we can prove

Proposition 1. Let $R$ be a reflexive binary relation on $A, p \in A$ and $T_{p}(R)$ defined by (3.1). Then $T_{p}(R)$ is a centred ternary relation on $A$ and its $p$-derived binary relation is just $R$.

Proof. It is evident that $T_{p}(R)$ is a ternary relation on $A$, its $p$-derived binary relation is just $R$ and $Z_{T_{p}(R)}(x, y) \supseteq\{y\} \neq \varnothing$ for all $x, y \in A$, i. e. $T_{p}(R)$ is centred.

In what follows, we focus on the relation between ternary relations preserving a given function and properties of assigned operations. 
Definition 7. Let $T$ be a ternary relation and $f$ an $m$-ary operation on $A$. We say that $f$ preserves $T$ if

$$
\begin{aligned}
\left(a_{1}, b_{1}, c_{1}\right), \ldots,\left(a_{m}, b_{m}, c_{m}\right) & \in T \text { implies } \\
& \left(f\left(a_{1}, \ldots, a_{m}\right), f\left(b_{1}, \ldots, b_{m}\right), f\left(c_{1}, \ldots, c_{m}\right)\right) \in T .
\end{aligned}
$$

It is worth noticing that the set of all operations on $A$ preserving a given relation $T$ forms a so-called clone. This topic is intensively investigated in contemporary algebra.

Definition 8. Let $f$ be an $m$-ary and $g$ an $n$-ary operation on $A$. We say that $f$ and $g$ commute with each other if

$$
\begin{aligned}
& f\left(g\left(x_{11}, \ldots, x_{1 n}\right), \ldots, g\left(x_{m 1}, \ldots, x_{m n}\right)\right) \\
& \quad=g\left(f\left(x_{11}, \ldots, x_{m 1}\right), \ldots, f\left(x_{1 n}, \ldots, x_{m n}\right)\right)
\end{aligned}
$$

for all $x_{11}, \ldots, x_{1 n}, \ldots, x_{m 1}, \ldots, x_{m n} \in A$.

We remark that also the set of all operations on $A$ commuting with a given operation $f$ forms a clone. Our next task is to compare both of these concepts.

Lemma 4. If $T$ is a centred ternary relation, $f$ an m-ary operation on $A$ commuting with a ternary operation $t$ assigned to $T$ then $f$ preserves $T$.

Proof. Let $t$ be a ternary operation assigned to $T$. Assume

$\left(a_{1}, b_{1}, c_{1}\right), \ldots,\left(a_{m}, b_{m}, c_{m}\right) \in T$. Let $f$ commute with $t$. Then $t\left(a_{i}, b_{i}, c_{i}\right)=b_{i}$ for $i=1, \ldots, m$ and hence

$$
\begin{aligned}
& t\left(f\left(a_{1}, \ldots, a_{m}\right), f\left(b_{1}, \ldots, b_{m}\right), f\left(c_{1}, \ldots, c_{m}\right)\right) \\
& =f\left(t\left(a_{1}, b_{1}, c_{1}\right), \ldots, t\left(a_{m}, b_{m}, c_{m}\right)\right) \\
& =f\left(b_{1}, \ldots, b_{m}\right)
\end{aligned}
$$

showing $\left(f\left(a_{1}, \ldots, a_{m}\right), f\left(b_{1}, \ldots, b_{m}\right), f\left(c_{1}, \ldots, c_{m}\right)\right) \in T$.

Clearly the sufficient condition used in the previous Lemma is not necessary. Such a condition is as follows.

Theorem 8. If $T$ is a centred ternary relation, $f$ an $m$-ary operation on $A$ and $t$ a ternary operation assigned to $T$ then $f$ preserves $T$ if and only if it satisfies the following identity:

$$
\begin{aligned}
& t\left(f\left(x_{1}, \ldots, x_{m}\right), f\left(t\left(x_{1}, y_{1}, z_{1}\right), \ldots, t\left(x_{m}, y_{m}, z_{m}\right)\right), f\left(z_{1}, \ldots, z_{m}\right)\right) \\
& \quad=f\left(t\left(x_{1}, y_{1}, z_{1}\right), \ldots, t\left(x_{m}, y_{m}, z_{m}\right)\right) .
\end{aligned}
$$

Proof. Assume that $f$ preserves $T$. Since $t$ is assigned to $T$ we have $\left(x_{i}, t\left(x_{i}, y_{i}, z_{i}\right), z_{i}\right) \in T$ for all $i=1, \ldots, m$. Hence

$$
\left(f\left(x_{1}, \ldots, x_{m}\right), f\left(t\left(x_{1}, y_{1}, z_{1}\right), \ldots, t\left(x_{m}, y_{m}, z_{m}\right)\right), f\left(z_{1}, \ldots, z_{m}\right)\right) \in T .
$$


Thus (3.2) holds.

Conversely, assume that $f$ satisfies (3.2) and $\left(a_{1}, b_{1}, c_{1}\right), \ldots,\left(a_{m}, b_{m}, c_{m}\right) \in T$. Then

for $i=1, \ldots, m$, and hence

$$
t\left(a_{i}, b_{i}, c_{i}\right)=b_{i}
$$

$$
\begin{aligned}
& t\left(f\left(a_{1}, \ldots, a_{m}\right), f\left(b_{1}, \ldots, b_{m}\right), f\left(c_{1}, \ldots, c_{m}\right)\right) \\
& =t\left(f\left(a_{1} \ldots, a_{m}\right), f\left(t\left(a_{1}, b_{1}, c_{1}\right), \ldots, t\left(a_{m}, b_{m}, c_{m}\right)\right), f\left(c_{1}, \ldots, c_{m}\right)\right) \\
& =f\left(t\left(a_{1}, b_{1}, c_{1}\right), \ldots, t\left(a_{m}, b_{m}, c_{m}\right)\right) \\
& =f\left(b_{1}, \ldots, b_{m}\right)
\end{aligned}
$$

proving $\left(f\left(a_{1}, \ldots, a_{m}\right), f\left(b_{1}, \ldots, b_{m}\right), f\left(c_{1}, \ldots, c_{m}\right)\right) \in T$. Hence, $f$ preserves $T$.

\section{Median-LiKe Algebras}

The concept of a median algebra was introduced by J. R. Isbell (see [5]) as follows: An algebra $\mathcal{A}=(A ; t)$ of type (3) is called a median algebra if it satisfies the following identities:

(M1) $t(x, x, y)=x$;

(M2) $t(x, y, z)=t(y, x, z)=t(y, z, x)$;

(M3) $t(t(x, y, z), v, w)=t(x, t(y, v, w), t(z, v, w))$.

It is well-known (see e.g. [1], [5]) that the ternary relation $T_{t}$ on $A$ assigned to $t$ via (2.1) is centred and, moreover, $\left|M_{T_{t}}(a, b, c)\right|=1$ for all $a, b, c \in A$. In fact, $t(a, b, c) \in M_{T_{t}}(a, b, c)$. In particular, having a distributive lattice $\mathscr{L}=(L ; \vee, \wedge)$ then $m(x, y, z)=M(x, y, z)$ and putting $t(x, y, z):=m(x, y, z)$, one obtains a median algebra. Conversely, every median algebra can be embedded into a distributive lattice. Moreover, the assigned ternary relation $T_{t}$ is the so-called "betweenness", see [10] and [11].

In what follows, we focus on the case when $M_{T}(a, b, c) \neq \varnothing$ for all $a, b, c \in A$ and $t(a, b, c) \in M_{T}(a, b, c)$ also in case $\left|M_{T}(a, b, c)\right| \geq 1$.

Definition 9. A median-like algebra is an algebra $(A ; t)$ of type (3) where $t$ satisfies (M1) and (M2) and where there exists a centred ternary relation $T$ on $A$ such that $t(x, y, z) \in M_{T}(x, y, z)$ for all $x, y, z \in A$.

Theorem 9. An algebra $\mathcal{A}=(A ; t)$ of type (3) is median-like if $t$ satisfies $(M 1)$, (M2) and

$$
t(x, t(x, y, z), y)=t(y, t(x, y, z), z)=t(z, t(x, y, z), x)=t(x, y, z) .
$$

Proof. If $T:=\left\{(x, y, z) \in A^{3} \mid t(x, y, z)=y\right\}$ then $t(x, y, z) \in M_{T}(x, y, z)$ for all $x, y, z \in A$.

Lemma 5. Every median algebra is a median-like algebra. 
Proof. As shown in [5], identities (M1), (M2), (M3) are equivalent to the identity

$$
t(x, t(x, z, w), t(y, z, w))=t(x, z, w) .
$$

Putting $w=y$ and using (M1) and (M2), we derive

$$
t(x, t(x, z, y), y)=t(x, t(x, z, y), t(y, z, y))=t(t(x, x, y), z, y)=t(x, z, y)
$$

whence (4.1) follows since according to (M2) we have $t(u, v, w)=t(x, y, z)$ for any permutation $(u, v, w)$ of $(x, y, z)$.

The following examples show that a median-like algebra need not be a median algebra.

Example 5. Put $A:=\{1,2,3,4,5\}$, let $t$ denote the ternary operation on $A$ defined by $t(x, x, y)=t(x, y, x)=t(y, x, x):=x$ for all $x, y \in A$ and $t(x, y, z):=$ $\min (x, y, z)$ for all $x, y, z \in A$ with $x \neq y \neq z \neq x$ and put $T:=\{(x, x, y) \mid x, y \in$ $A\} \cup\{(y, x, x) \mid x, y \in A\} \cup\left\{(x, y, z) \in A^{3} \mid y<x<z\right\} \cup\left\{(x, y, z) \in A^{3} \mid y<z<x\right\}$. Then $t$ satisfies (M1) and (M2) and $t(x, y, z) \in M_{T}(x, y, z)$ for all $x, y, z \in A$. This shows that $(A ; t)$ is median-like. However, this algebra is not a median algebra since

$$
t(t(1,3,4), 2,5)=t(1,2,5)=1 \neq 2=t(1,2,2)=t(1, t(3,2,5), t(4,2,5))
$$

and hence (M3) is not satisfied.

Example 6. Consider the lattice $M_{3}$ given in FIGURE 1 below.

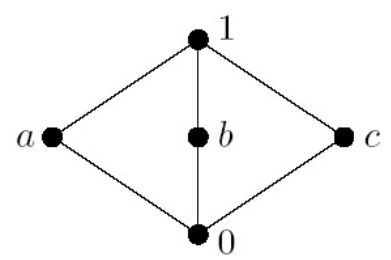

FIGURE 1.

Then $M_{3}$ is not distributive, $m(a, b, c)=0$ and $M(a, b, c)=1$. Define $(x, y, z) \in T$ if and only if $y \in[x \wedge z, x \vee z]$. Let $t$ be an assigned operation defined as follows

$$
t(x, y, z):=m(x, y, z) .
$$

Then $t(x, y, z) \in M_{T}(x, y, z)$ for all triples of elements $x, y, z$ and hence $\left(M_{3} ; t\right)$ is a median-like algebra. However, it is not a median algebra because identity (M3) is violated:

$$
t(t(a, b, c), a, 1)=t(0, a, 1)=a \neq 1=t(a, 1,1)=t(a, t(b, a, 1), t(c, a, 1)) .
$$

The previous example motivated us to state a general construction for lattices which need not be neither distributive nor modular. 
Theorem 10. Let $\mathscr{L}=(L ; \vee, \wedge)$ be a lattice. Define $t_{1}(x, y, z):=m(x, y, z)$, $t_{2}(x, y, z):=M(x, y, z)$. Then $\mathcal{A}_{1}:=\left(L ; t_{1}\right)$ and $\mathcal{A}_{2}:=\left(L ; t_{2}\right)$ are median-like algebras. Moreover, the following conditions are equivalent

(a) $\mathscr{A}_{1}=\mathscr{A}_{2}$;

(b) $\mathcal{A}_{1}$ is a median algebra;

(c) $\mathscr{L}$ is distributive.

Proof. Since both $m(x, y, z)$ and $M(x, y, z)$ satisfy (M1) and (M2) and $m(x, y, z), M(x, y, z) \in[m(x, y, z), M(x, y, z)]=M_{T}(x, y, z)$ for $(x, y, z) \in L^{3}$ and $T:=\left\{(x, y, z) \in L^{3} \mid x \wedge z \leq y \leq x \vee z\right\}, \mathcal{A}_{1}, \mathcal{A}_{2}$ are median-like algebras. It is well-known that $m(x, y, z)=M(x, y, z)$ if and only if $\mathscr{L}$ is distributive which proves $(a) \Leftrightarrow(c)$. The implication $(c) \Rightarrow(b)$ is well-known (see e.g. [1], [5]). Finally, we prove $(b) \Rightarrow(c)$. Assume that (b) holds but (c) does not. Then $\mathscr{L}$ contains either $\mathcal{M}_{3}=(\{0, a, b, c, 1\} ; \vee, \wedge)$ or $\mathcal{N}_{5}=(\{0, a, b, c, 1\} ; \vee, \wedge)($ with $a<c)$ as a sublattice. In the first case we have

$$
t(t(a, b, c), a, 1)=t(0, a, 1)=a \neq 1=t(a, 1,1)=t(a, t(b, a, 1), t(c, a, 1))
$$

whereas in the second case

$$
t(t(c, b, a), a, 1)=t(a, a, 1)=a \neq c=t(c, 1, a)=t(c, t(b, a, 1), t(a, a, 1))
$$

which shows that (M3) does not hold. This is a contradiction to (b). Hence (c) holds.

Comparing our definition with Theorem 2, we conclude:

Corollary 2. An algebra ( $A ; t)$ of type (3) is median-like if $t$ satisfies (M2) and if it is assigned to a centred antisymmetric or non-sharp ternary relation on A.

Let us mention that median-like algebras form a variety because they are defined by identities. Moreover, this variety is congruence distributive, i. e. Con $\mathcal{A}$ is distributive for every median-like algebra $\mathcal{A}$, because the operation $t$ is a majority term, i. e. it satisfies by (M1) and (M2)

$$
t(x, x, y)=t(x, y, x)=t(y, x, x)=x .
$$

Theorem 11. let $\mathscr{L}=(L ; \vee, \wedge)$ be a lattice and t a ternary operation on $L$ satisfying $(M 1)$ and $(M 2)$ and $t(x, y, z) \in[m(x, y, z), M(x, y, z)]$ for all $x, y, z \in A$. Then $\mathcal{A}:=(L ; t)$ is a median-like algebra.

Proof. Put $T:=\left\{(x, y, z) \in L^{3} \mid x \wedge z \leq y \leq x \vee z\right\}$. Then $M_{T}(a, b, c)$ $=[m(a, b, c), M(a, b, c)]$ for all $a, b, c \in L$. Hence $t(a, b, c) \in M_{T}(a, b, c)$ for all $a, b, c \in L$ showing that $\mathcal{A}$ is a median-like algebra. 


\section{CyClic Algebras}

Apart from the "betweenness" relation, another ternary relation plays an important role in mathematics. It is the so-called cyclic order, see e.g. [4], [9] and references there.

Definition 10. A ternary relation $T$ on $A$ is called asymmetric if

$$
(a, b, c) \in T \text { for } a \neq b \neq c \text { implies } \quad(c, b, a) \notin T .
$$

A ternary relation $C$ on $A$ is called a cyclic order if it is cyclic, asymmetric, cyclically transitive and satisfies $(a, a, a) \in C$ for each $a \in A$.

Remark 4. Let $C$ be a cyclic order on a set $A$. Then $(a, b, a) \notin C$ for all $a, b \in A$ with $a \neq b$. Namely, if $(a, b, a) \in C$ then, by (5.1), $(a, b, a) \notin C$, a contradiction. Since $C$ is cyclic, we have also $(a, a, b),(b, a, a) \notin C$.

Applying (5.1), we derive immediately

Lemma 6. A centred ternary relation $T$ on $A$ is asymmetric if and only if any assigned ternary operation $t$ satisfies the implication:

$$
(t(x, y, z)=y \text { and } x \neq y \neq z) \quad \Longrightarrow \quad t(z, y, x) \neq y .
$$

Similarly as for "betweenness" relations, we can derive an algebra of type (3) for a centred cyclic order by means of its assigned operation.

Definition 11. A cyclic algebra is an algebra assigned to a cyclic relation.

Cyclic algebras can be characterized by certain identities and the implication (5.2) as follows.

Theorem 12. An algebra $\mathcal{A}=(A ; t)$ of type (3) is a cyclic algebra if and only if it satisfies (5.2) and

$$
\begin{aligned}
& t(x, t(x, y, z), z)=t(x, y, z), \\
& t(t(x, y, z), z, x)=z, \\
& t(x, t(x, y, t(x, z, u)), u)=t(x, y, t(x, z, u)), \\
& t(x, x, x)=x .
\end{aligned}
$$

Proof. Assume that $\mathcal{A}=(A ; t)$ satisfies the above identities and (5.2). By Theorem 1 and the first identity, $t$ is an assigned operation of a certain centred ternary relation $C$ on $A$. By Theorem 2 and the second and third identity, $C$ is cyclic and cyclically transitive. The fourth identity gets $(x, x, x) \in C$ for each $x \in A$. Finally, Lemma 6 yields that $C$ is asymmetric and hence a cyclic order on $A$. Of course, $t$ is an assigned operation of $C$ and hence $\mathcal{A}=(A ; t)$ is a cyclic algebra.

The converse follows directly by Definition 11 . 


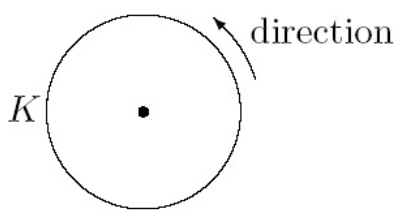

FIGURE 2.

Example 7. Let $K$ be a circle in a plane with a given direction, see FIGURE 2. Define a ternary relation $C$ on $K$ as follows:

$$
\begin{aligned}
& (a, a, a) \in C \text { for each } a \in K \text { and } \\
& (a, b, c) \in C \text { if } a \rightarrow b \text { and } b \rightarrow c \text { for } a \neq b \neq c .
\end{aligned}
$$

It is an easy exercise to check that $C$ is a cyclic order on $K$. If $a, b \in K$ then either $a=b$ and hence $Z_{C}(a, a)=\{a\}$ or $a \neq b$ thus $Z_{C}(a, b)$ equals the arc of $K$ between $a$ and $b$, i. e. it contains a continuum of points. Hence $C$ is centred. For any assigned operation $t$, the algebra $\mathcal{A}(C)=(K ; t)$ is a cyclic algebra.

\section{REFERENCES}

[1] H.-J. Bandelt and J. Hedlikova, "Median algebras," Discrete Math., vol. 45, pp. 1-30, 1983.

[2] I. Chajda and H. Länger, "Quotients and homomorphisms of relational systems," Acta Univ. Palacki. Olomuc., Fac. Rerum Nat., Math., vol. 49, no. 2, pp. 37-47, 2010.

[3] I. Chajda and H. Länger, "Groupoids assigned to relational systems," Math. Bohem., vol. 138, no. 1, pp. 15-23, 2013.

[4] I. Chajda and V. Novák, "On extensions of cyclic orders," Čas. Pěstování Mat., vol. 110, pp. 116-121, 1985.

[5] J. R. Isbell, "Median algebra," Trans. Am. Math. Soc., vol. 260, pp. 319-362, 1980.

[6] J. Ježek and R. Quackenbush, "Directoids: Algebraic models of up-directed sets," Algebra Univers, vol. 27, no. 1, pp. 49-69, 1990.

[7] N. Megiddo, "Partial and complete cyclic orders," Bull. Am. Math. Soc., vol. 82, pp. 274-276, 1976.

[8] G. Müller, "Lineare und zyklische ordnung,” Prax. Math., vol. 16, pp. 261-269, 1974.

[9] V. Novak, "Cyclically ordered sets," Czech. Math. J., vol. 32, pp. 460-473, 1982.

[10] E. Pitcher and M. F. Smiley, "Transitivities of betweenness," Trans. Am. Math. Soc., vol. 52, pp. 95-114, 1942.

[11] M. Sholander, “Medians and betweenness," Proc. Am. Math. Soc., vol. 5, pp. 801-807, 1954.

Authors' addresses

\section{Ivan Chajda}

Palacký University Olomouc, Faculty of Science, Department of Algebra and Geometry, Třída 17. listopadu 12, 77146 Olomouc, Czech Republic

E-mail address: ivan.chajda@upol.cz 
Miroslav Kolařík

Palacký University Olomouc, Faculty of Science, Department of Computer Science, Tř́ída 17. listopadu 12, 77146 Olomouc, Czech Republic

E-mail address: miroslav.kolarik@upol.cz

\section{Helmut Länger}

Vienna University of Technology, Institute of Discrete Mathematics and Geometry, Wiedner Hauptstraße 8-10, 1040 Vienna, Austria

E-mail address: h.laenger@tuwien.ac.at 